\title{
Metasurface-Based THz Dual-Band Absorber Sensor for the Measurement of Refractive Index Variations of Chemical and Biological Substances ${ }^{\dagger}$
}

\author{
Mohammed Janneh 1, Andrea De Marcellis 1,*, Elia Palange ${ }^{1}$, Ayodya T. Tenggara ${ }^{2}$ and \\ Doyoung Byun ${ }^{2}$ \\ 1 Department of Industrial and Information Engineering and Economics, University of L'Aquila, \\ 67100 L'Aquila AQ, Italy; mohammed.janneh1@graduate.univaq.it (M.J.); elia.palange@univaq.it (E.P.) \\ 2 Department of Mechanical Engineering, Sungkyunkwan University, Seoul, Korea; \\ ayodya.ugm05@gmail.com (A.T.T.); dybyun@skku.edu (D.B.) \\ * Correspondence: andrea.demarcellis@univaq.it; Tel.: +39-0862-43-4424 \\ + Presented at the Eurosensors 2017 Conference, Paris, France, 3-6 September 2017.
}

Published: 16 August 2017

\begin{abstract}
In this paper we report on the results of the simulations of the electromagnetic properties of a metasurface composed of a 2D-array of plasmonic nanoantennas acting as polarisation insensitive dual band $\mathrm{THz}$ perfect absorber able to achieve absorption coefficients and quality factors up to $99.6 \%$ and 122 , respectively. The 2D-array is designed on top of a polyimide flexible film separating it from an Ag ground layer. The plasmonic nanoantenna is composed of two Ag quadrangular rings and an $\mathrm{Ag}$ diamond-shape dot is placed at their geometrical centre. The absorption resonances show a linear frequency shift with respect to variations of the refractive index of transparent substances adsorbed on the metasurface. This allows the dual-band absorber to be employed as a sensor of the chemical/physical modifications taking place on the substance. In particular, in terms of the refractive index units, the sensor based on the dual-band absorber can achieve sensitivities up to $310 \mathrm{GHz} / \mathrm{RIU}$.
\end{abstract}

Keywords: THz perfect absorber; functionalised metasurfaces; plasmonic sensors

\section{Introduction}

The study of the electromagnetic (e.m.) properties of MetaSurfaces (MS) used to implement perfect $\mathrm{THz}$ absorbers is attracting great attention for their potential applications as imagers and high-sensitive detectors as well as chemical, drug and explosive substance sensors [1-3]. In general, MS are 2D-arrays of ordered metal sub-wavelength NanoAntennas (NA) grown on dielectric, semiconductor and/or flexible plastic substrates. The NA shapes, sizes and mutual distances determine the MS spectral behaviour in terms of frequency and polarisation dependent reflection and transmission responses [4]. In particular, for the performance optimisation of the perfect $\mathrm{THz}$ absorbers used as sensing devices, it is necessary to study NA geometries and 2D-array structures with an e.m. response insensitive to the polarisation of the incident radiation and characterised by narrow spectral bandwidth, high quality factors (Q-factors) and absorption coefficients approaching $100 \%[5,6]$. In this regard, the main difficulty for the enhancement of the MS-based device sensitivity and resolution is the relatively low value of the achieved Q-factors that limits the detection of extremely small MS resonance frequency shifts occurring in presence of substances close-to or adsorbed-on the MS. To date, the maximum reported Q-factors for a perfect dual-band absorber in the $\mathrm{THz}$ region is equal to 59.8 achieved by using a simple metal rod shape for the NA with a maximum absorption coefficient of $99.6 \%$ [6]. However, the e.m. properties of this particular MS are intrinsically polarisation dependent. On the other hand, the polarisation independent version makes 
use of cross-shaped NA for which a Q-factor equal to 48.8 has been achieved with a maximum absorption coefficient equal to $85.3 \%$ [6]. Moreover, for the dual-band perfect absorber configurations it is observed great changes of the Q-factors of the two absorption resonances. In the case of the results reported in [6], for example, the Q-factors of the absorption resonances occurring at the higher frequency are 8.4 and 7.7, smaller than those ones related to the lower frequency absorption resonance for the rod- and cross-shaped NA, respectively. As a consequence, only one of the two absorption resonances of the dual-band perfect absorbers (i.e., that one with the highest Q-factor) can be properly used to probe the presence of a substance (i.e., an analyte) adsorbed on its surface. In this paper, we present a novel $\mathrm{THz}$ dual-band perfect absorber that overcomes this problem allowing to reach Qfactors higher than 100 for both the two absorption resonances.

\section{Design, Results and Discussion}

In Figure 1 is reported the cross section (panel a) and the plane view (panel b) of the proposed dual-band perfect absorber. The cross section shows that the MS 2D-array of Ag NA is located on top of a polyimide flexible film that separates the MS from the Ag ground layer. Here $t_{m}, t_{s}$ and $t$ are the Ag MS, polyimide spacer and Ag ground layer thicknesses, respectively. The use of the polyimide film allows fabricating the MS by using, for instance, the electro-hydrodynamic jet printing technique [7]. Referring now to the plane view reported in panel (b) of Figure 1, the NA geometry is composed of two concentric Ag square rings and of an Ag diamond-shape dot located at their centre. In the same figure, the lengths $L_{x}, L_{1}, L_{2}$ and $L_{3}$ are the outer and inner sides of the outer and inner square rings, respectively, and $L_{4}$ is the diagonal of the diamond-shape dot. Note that $L_{x}$ represents also the lattice parameter of the 2D-array MS.

The e.m. response of the designed MS has been investigated by using FEM analysis in the frequency domain. The simulations were performed illuminating the MS by using a TM and TE linear polarised e.m. plane wave propagating along the $z$-direction perpendicular to the MS $(x, y)$ plane (see Figure 1). Starting from the unit cell reported in Figure 1, the MS 2D-array was obtained by using periodic boundary conditions along the $x$ - and $y$-directions and scattering boundary conditions along the $z$-direction. Under these conditions, the symmetry properties of the NA shape allow the e.m. response of MS to be independent from the polarisation of the incident radiation. For the numerical simulations the following values of the dual-band perfect absorber geometrical parameters have been used: $L_{x}=100 \mu \mathrm{m}, L_{1}=85 \mu \mathrm{m}, L_{2}=70 \mu \mathrm{m}, L_{3}=50 \mu \mathrm{m}, L_{4}=21.3 \mu \mathrm{m}, t_{m}=0.2 \mu \mathrm{m}, t_{s}=8 \mu \mathrm{m}, t=0.4 \mu \mathrm{m}$. Moreover, the Ag relative electric permittivity has been calculated through the Drude model using the plasma frequency $\omega_{p}=1.4 \times 10^{16} \mathrm{rad} / \mathrm{s}$ and the damping coefficient $\Gamma_{A g}=0.32 \times 10^{14} \mathrm{~s}^{-1}$. The polyimide dielectric permittivity equal to $(3.6+0.005 \mathrm{i})$ is assumed constant within the frequency range chosen for the simulations. Since the MS transmission is eliminated, due to the presence of the $\mathrm{Ag}$ ground plane in the unit cell which is much thicker than the Ag penetration depth in the $\mathrm{THz}$ spectrum range, the MS absorption response can be calculated by using the relation $A(\omega)=1-R(\omega)$, where $A(\omega)$ and $R(\omega)$ are the frequency dependent absorption and reflection coefficients, respectively.

In panel (a) of Figure 2 is reported the spectral response of the dual-band perfect absorber for the TM polarisation mode showing two resonances centred at $f_{1}=1.80 \mathrm{THz}$ and $f_{2}=2.27 \mathrm{THz}$ both having maximum absorption coefficients and Q-factors equal to $A\left(f_{1}\right)=A\left(f_{2}\right)=99.6 \%$ and $Q\left(f_{1}\right)=122$ and $Q\left(f_{2}\right)=96$, respectively. Identical results have been obtained for the TE polarisation mode. The values of the Q-factors indicate that the designed dual-band absorber accomplishes a strong frequency selectivity for both the frequencies $f_{1}$ and $f_{2}$ demonstrating an overall improvement more than a factor 2 with respect to the best results reported in literature [6,8-10]. In order to explain the physical origins of the two resonances of the dual-band absorber, in panel (b) of Figure 2 are reported its spectral response under the following conditions: (i) if the NA inner square ring is absent, only the resonance centred at $f_{1}$ is present (continuous black line) with $Q^{\prime}\left(f_{1}\right)=121$ and $A^{\prime}\left(f_{1}\right)=58 \%$; (ii) if the outer NA square ring is removed, only the resonance at $f_{2}$ is observed (red dotted line) with $Q^{\prime}\left(f_{2}\right)=$ 106 and $A^{\prime}\left(f_{2}\right)=98 \%$, thus similar to the corresponding one shown in panel (a) of Figure 1 These results demonstrate that the $\mathrm{Q}$-factors are not affected by the absence of one of the two NA rings and 
that the resonance at the frequency $f_{1}$ is strongly enhanced through the e.m. interactions arising in the region between the two NA square rings. In order to prove this, in Figure 3 we report the distributions of the intensity of the electric field just above the MS calculated at the two frequencies $f_{1}$ and $f_{2}$.

The interesting e.m. properties of the proposed dual-band absorber suggested to use it as a sensor to measure the changes of the refractive index of a transparent analyte adsorbed on top of the MS. In panel (a) of Figure 4 we report the dependence of the frequency resonances $f_{1}$ and $f_{2}$ on the variation of the analyte refractive index. The linear dependence allows defining the sensitivity of the sensor based on the dual-band absorber that, in terms of the Refractive Index Unit (RIU), reaches values up to $\sigma\left(f_{1}\right)=180 \mathrm{GHz} / \mathrm{RIU}$ and $\sigma\left(f_{2}\right)=310 \mathrm{GHz} / \mathrm{RIU}$ for the resonance frequencies $f_{1}$ and $f_{2}$, respectively (see panel (b) of Figure 4). Finally, the plot in panel (b) of Figure 4 shows that the sensitivity depends on the analyte thickness opening the possibility to use the designed dual-band absorber to measure also the amount of analyte adsorbed on the MS.
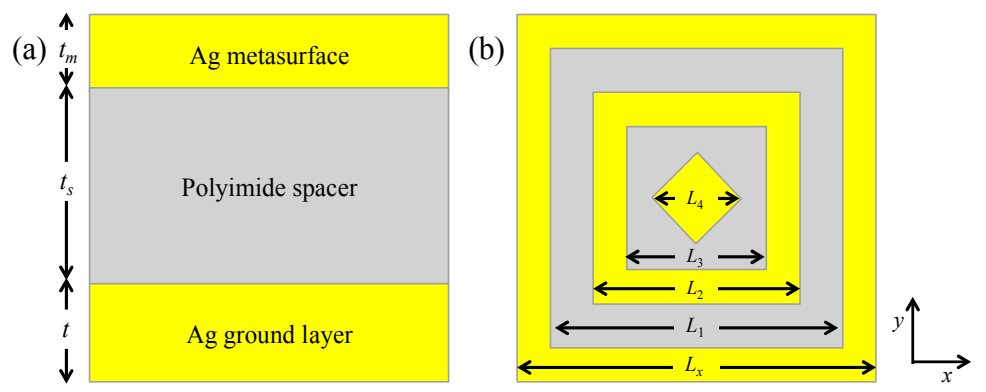

Figure 1. Panel (a): the cross section of the THz dual-band absorber where $t_{m}, t_{s}$ and $t$ are the Ag metasurface, polyimide spacer and Ag ground layer thicknesses, respectively. Panel (b): the metasurface plasmonic nanoantenna plane view.
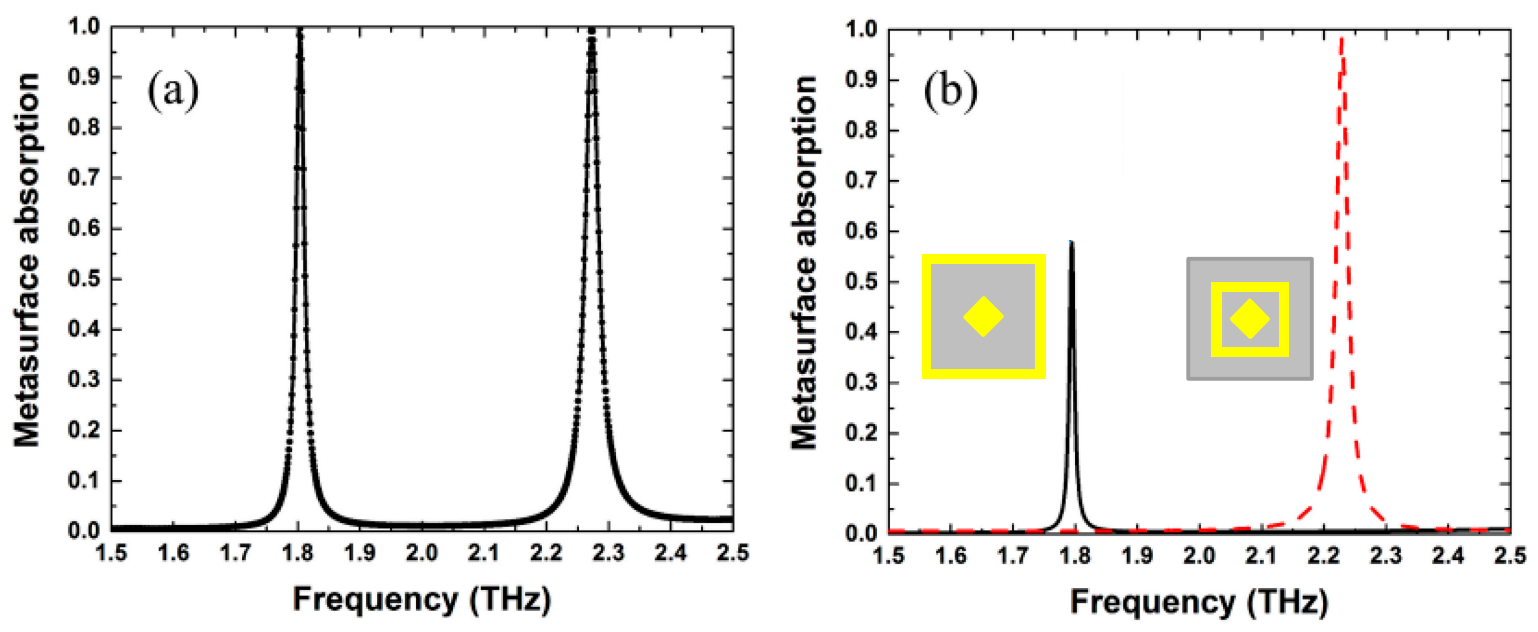

Figure 2. Panel (a): the polarisation insensitive spectral response of the dual-band absorber showing the two resonances at $f_{1}=1.80 \mathrm{THz}$ and $f_{2}=2.27 \mathrm{THz}$. Panel (b): the e.m. response of the dual-band absorber in absence of the NA inner ring (black continuous line) or of the NA outer ring (red dot line). 

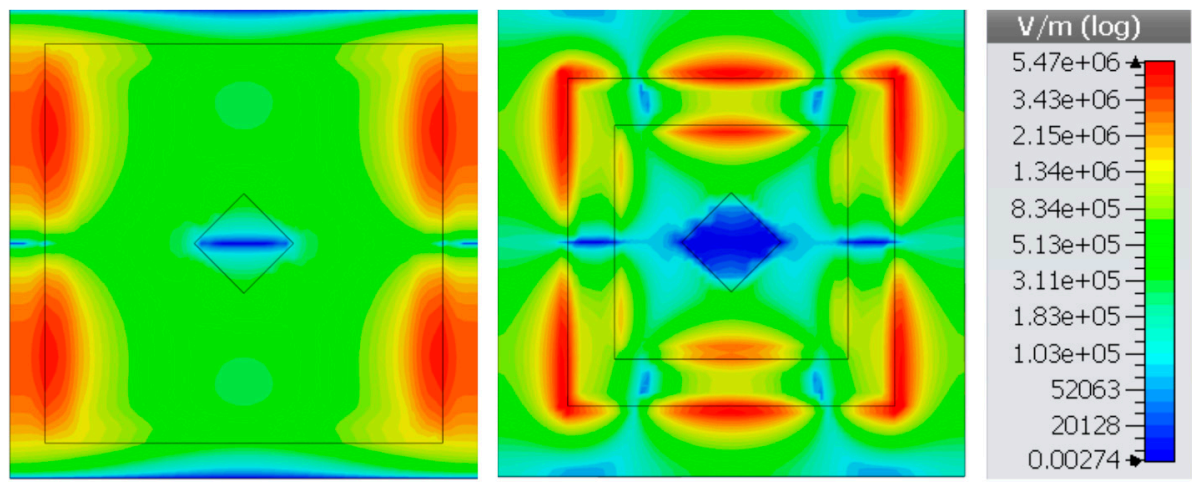

Figure 3. The distributions of the intensity of the electric field calculated just above the MS for the resonance frequencies $f_{1}$ without the inner ring (left) and $f_{2}$ without the outer ring (right).
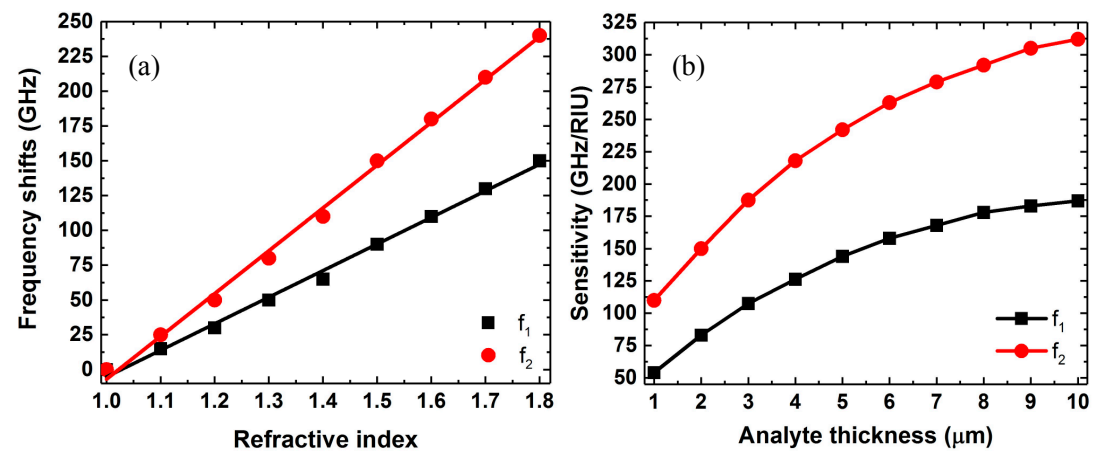

Figure 4. Panel (a): the frequency shift as a function of the refractive index of a $5 \mu \mathrm{m}$ thick analyte adsorbed on the MS. Panel (b): the dependence of the sensitivity respect to the variation of the analyte thickness for a refractive index equal to 1.8 .

\section{Conclusions}

In this paper we reported on the electromagnetic response of a novel polarisation insensitive dual-band perfect absorber composed of a 2D-array of plasmonic nanoantennas able to achieve absorption coefficients and quality factors up to $99.6 \%$ and 122, respectively. With respect to the previous results reported in the literature, the proposed metasurface geometry allows having the same properties in terms of absorption coefficient and quality factor for both the two absorption resonances. This permits to employ the dual-band absorber as a sensor of chemical/physical modifications of analyte adsorbed on the metasurface. In particular, in terms of the refractive index units, the sensor based on the dual-band absorber can achieve sensitivities up to $310 \mathrm{GHz} / \mathrm{RIU}$.

Acknowledgments: $\mathrm{ADM}$ and EP acknowledge the partial financial support of Regione AbruzzoNANOPREPAINT project-PAR FSC ABRUZZO 2007-2013, Linea di Azione I.1.1.A.

Conflicts of Interest: The authors declare no conflict of interest.

\section{References}

1. Jamali, A.A.; Witzigmann, B. Plasmonic perfect absorbers for biosensing applications. Plasmonics 2014, 9, 1265-1270.

2. Chen, T.; Li, S.; Sun, H. Metamaterials Application in Sensing. Sensors 2012, 12, 2742-2765.

3. Federici, J.F.; Schulkin, B.; Huang, F.; Gary, D.; Barat, R.; Oliveira, F.; Zimdars, D. THz imaging and sensing for security applications-explosives, weapons, and drugs. Semicond. Sci. Technol. 2005, 20, S266-S280.

4. De Marcellis, A.; Palange, E.; Janneh, M.; Rizza, C.; Ciattoni, A.; Mengali, S. Design Optimisation of Plasmonic Metasurfaces for Mid-Infrared High-Sensitivity Chemical Sensing. Plasmonics 2017, 12, $293-298$.

5. Wen, Q.Y.; Zhang, H.W.; Xie, Y.S.; Yang, Q.H.; Liu, Y.L. Dual band terahertz metamaterial absorber: Design, fabrication, and characterization. Appl. Phys. Lett. 2009, 95, 241111-1-241111-3. 
6. Wang, X.B.; Zhai, X.; Wang, Z.C.; Huang, Q.W.; Wang, L.L. A novel dual-band terahertz metamaterial absorber for a sensor application. J. Appl. Phys. 2015, 117, 014504-1-014504-5.

7. Tenggara, A.P.; Park, S.J.; Yudistira, H.T.; Ahn, Y.H.; Byun, D. Fabrication of terahertz metamaterials using electrohydrodynamic jet printing for sensitive detection of yeast. J. Micromech. Microeng. 2017, 27, 0350091-035009-8.

8. Grant, J.; Ma, Y.; Saha, S.C.; Lok, L.B.; Khalid, A.; Cumming, D.R.S. Polarization insensitive terahertz metamaterial absorber. Opt. Lett. 2011, 36, 1524-1526.

9. Chen, X.; Fan, W. Ultra-flexible polarization-insensitive multiband terahertz metamaterial absorber. Appl. Opt. 2015, 54, 2376-2382.

10. Yan, X.; Liang, L.J.; Ding, X.; Yao, J.Q. Solid analyte and aqueous solutions sensing based on a flexible terahertz dual-band metamaterial absorber. Opt. Eng. 2017, 56, 027104-1-027104-6.

(C) 2017 by the authors. Licensee MDPI, Basel, Switzerland. This article is an open access article distributed under the terms and conditions of the Creative Commons Attribution (CC BY) license (http://creativecommons.org/licenses/by/4.0/). 